\title{
Medication Adherence in Korean Hypertensive Patients
}

\author{
Young Gyu Cho* \\ Department of Family Medicine, Inje University Seoul Paik Hospital, Inje University College of Medicine, Seoul, Korea
}

\section{See original paper on 325}

Hypertension is associated with increased cardiovascular disease (CVD) risk and contributes to morbidity and mortality from CVD. Hypertension guidelines recommend lifestyle interventions and drug treatment to lower blood pressure. Most hypertensive patients require drug treatment to achieve their target blood pressure. ${ }^{1)}$ The Korea National Health and Nutrition Examination Survey, 2016 shows that the awareness, treatment, and control rates of hypertension among all Korean hypertensive patients aged 30 years or older were $68.9 \%$, $65.0 \%$, and $46.5 \%$, respectively. Even among patients treated with antihypertensive medications, about $30 \%$ could not control blood pressure to a level of $<140 / 90 \mathrm{~mm} \mathrm{Hg} .{ }^{2)}$ Poor adherence to prescribed medications is thought to be the most important cause of insufficient blood pressure control. The appropriate antihypertensive medication adherence rate was only $54.7 \%$ among beneficiaries of National Health Insurance in Korea. ${ }^{3)}$

Adherence is defined by the World Health Organization as the extent to which a person's behavior-medication intake, diet adherence, and/or executing lifestyle changes-corresponds with the agreed-upon recommendations from a health care provider. ${ }^{4)}$ Adherence is distinguished from compliance in that it emphasizes the patient's agreement to the recommendations. Medication adherence is influenced by patient beliefs about disease and medicine. The health belief model (HBM) is a psychological framework developed to explain and predict health behavior. Hypertensive patients will take prescribed medicines if they believe that they are susceptible to complications of hypertension (perceived susceptibility), if they believe that those complications would be serious (perceived severity), if they believe that prescribed medicines would reduce the susceptibility and/or severity of those complications (perceived benefits), and if they believe that taking the medicines would not lead to negative attributes (perceived barriers). Cues for medicine intake and self-efficacy are also factors in medication adherence in hypertensive patients. ${ }^{5)}$ Various modifying factors including age, sex, personality, socioeconomic status, knowledge about hypertension, and duration of treatment can indirectly influence adherence by affecting the components of HBM. Yue et al. ${ }^{6}$ applied HBM to explain antihypertensive medication adherence among Chinese patients. They reported that HBM explained $48.8 \%$ of the variance in antihypertensive medication adherence, with an overall predictive accuracy of $\mathbf{8 2 . 8 \%}$. In their study, older age, longer duration of hypertension, longer duration of drug use, and combined use with antiplatelet agents were significantly associated with better antihypertensive medication adherence and affected the components of HBM.

In the present issue, Choi et al. ${ }^{7}$ investigated factors affecting adherence to antihypertensive medication in Korean patients. The study subjects consisted of 1,523 Korean hypertensive patients who visited family physicians. The study was conducted in 24 facilities (two primary care clinics and 22 level 2 or 3 hospitals) located in urban and metropolitan areas. They reported that age $\geq 65$ years, concomitant medication for diabetes, a family history of hypertension and/or CVD, being on $\geq 2$ classes of antihypertensive medications, high frequency of exercise, and treatment in a metropolitan-located hospital were associated with good adherence, but high salt intake was associated with poor adherence. Older age, comorbidities with other cardiovascular risk factors, and family history of CVD may result in good medication adherence by affecting perceived susceptibility and severity of hypertension complications. The number of antihypertensive drugs and the dosing frequency are con- 
sidered to adversely affect medication adherence, and simplification of the drug regimen is regarded as an effective intervention to improve adherence. ${ }^{8)}$ However, Choi et al. ${ }^{7)}$ reported that patients taking multiple classes of antihypertensive medications had good adherence. They categorized patients' drug regimens based on the number of classes of antihypertensive medication, not based on the number of pills. Various combination pills containing two antihypertensive agents with different action modes have been developed and used. The number of classes of antihypertensive medication may simply reflect the severity of hypertension, if the number of prescribed pills is the same. Adherent patients are more likely to follow all preventive recommendations to improve their health. ${ }^{9)}$ This 'healthy behavior' concept can explain the finding that high frequencies of exercise were associated with good medication adherence but high salt intake was associated with poor medication adherence in the study by Choi et al. ${ }^{7)}$

Continuity of care is known to be associated with medication adherence. Jung and Byeon ${ }^{10)}$ reported that having a usual source of care was associated with less non-adherence to medication in Korean patients with chronic diseases. The Korean government introduced the chronic disease management program (CDMP), a multi-level intervention including copayment reduction and physician incentives in 2012. The aim of CDMP was to improve blood pressure and glycemic control in patients with hypertension and type 2 diabetes by strengthening the function of clinics as primary care institutions. Kim et al. ${ }^{11)}$ found that CDMP improved medication adherence in patients with hypertension. In order to promote adherence to antihypertensive medication, policies and strategies to strengthen primary care and improve continuity of care should be developed and implemented consistently.

\section{CONFLICT OF INTEREST}

No potential conflict of interest relevant to this article was reported.

\section{REFERENCES}

1. Williams B, Mancia G, Spiering W, Agabiti Rosei E, Azizi M, Burnier M, et al. $2018 \mathrm{ESC} / \mathrm{ESH}$ guidelines for the management of arterial hypertension. Eur Heart J 2018;39:3021-104.

2. Ministry of Health and Welfare, Korea Centers for Disease Control and Prevention. Korea health statistics 2016: Korea National Health and Nutrition Examination Survey (KNHANES VII-1). Cheongju: Korea Centers for Disease Control and Prevention; 2017.

3. Park JH. Antihypertensive drug medication adherence of National Health Insurance beneficiaries and its affecting factors in Korea [dissertation]. Seoul: Seoul National University; 2006.

4. World Health Organization. Adherence to long-term therapies: evidence for action. Geneva: World Health Organization; 2003.

5. Jones CL, Jensen JD, Scherr CL, Brown NR, Christy K, Weaver J. The health belief model as an explanatory framework in communication research: exploring parallel, serial, and moderated mediation. Health Commun 2015;30:566-76.

6. Yue Z, Li C, Weilin Q, Bin W. Application of the health belief model to improve the understanding of antihypertensive medication adherence among Chinese patients. Patient Educ Couns 2015;98:669-73.

7. Choi HY, Oh IJ, Lee JA, Lim J, Kim YS, Jeon TH, et al. Factors affecting adherence to antihypertensive medication. Korean J Fam Med 2018;39:325-32.

8. Elliott WJ. Improving outcomes in hypertensive patients: focus on adherence and persistence with antihypertensive therapy. J Clin Hypertens (Greenwich) 2009;11:376-82.

9. Burnier M. Drug adherence in hypertension. Pharmacol Res 2017;125(Pt B):142-9.

10. Jung Y, Byeon J. The association between having a usual source of care and adherence to medicines in patients with chronic diseases. Korean J Clin Pharm 2016;26:128-36.

11. Kim JA, Kim ES, Lee EK. Evaluation of the chronic disease management program for appropriateness of medication adherence and persistence in hypertension and type-2 diabetes patients in Korea. Medicine (Baltimore) 2017;96:e6577. 